\title{
Short- and Long-Term Exposure to Particulate Matter and Pulse Wave Velocity
}

\author{
Young Jun Park', Yu Jin Cho', Jinseul Kwak², Youn-Hee Lim³, Minseon Park',** \\ 'Department of Family Medicine, Seoul National University Hospital, Seoul National University College of Medicine, Seoul, Korea \\ ${ }^{2}$ Department of Biomedical Engineering, Seoul National University College of Medicine, Seoul, Korea \\ ${ }^{3}$ Section of Environmental Health, Department of Public Health, Faculty of Health and Medical Sciences, University of Copenhagen, Copenhagen, \\ Denmark
}

Background: In hemodialysis patients, brachial-ankle pulse wave velocity (baPWV) levels are affected by particulate matter with an aerodynamic diameter of $10 \mu \mathrm{m}$ or less $\left(\mathrm{PM}_{10}\right)$. We conducted this study to determine whether there is an association between short- and long-term $\mathrm{PM}_{10}$ exposure and baPWV in apparently healthy adults aged 40 years and older.

Methods: A total of 1,628 subjects who underwent health examinations between 2006 and 2009 were included in the study. On the basis of the day of medical screening, the 1-3-day and 365-day moving averages of $\mathrm{PM}_{10}$ concentrations were used to evaluate the association between short- and long-term exposure to $\mathrm{PM}_{10}$ and high baPWV ( $\geq$ the third quartile of baPWV, 1,534 cm/s) using logistic regression models. Additional subgroup analyses were conducted according to age, sex, obesity (body mass index $\geq 25.0 \mathrm{~kg} / \mathrm{m}^{2}$ ), and comorbidities such as metabolic syndrome.

Results: No statistically significant associations were identified between short-term and long-term exposure to $\mathrm{PM}_{10}$ and baPWV in any of the subjects and subgroups. A 10- $\mu \mathrm{g} / \mathrm{m}^{3}$ increase in the 2-day moving average of $\mathrm{PM}_{10}$ exposure was marginally associated with high baPWV in non-obese subjects (odds ratio, $1.059 ; \mathrm{P}=0.058$ ). This association in non-obese subjects was significantly different from that in obese subjects $(\mathrm{P}=0.038)$.

Conclusion: This study did not show statistically significant associations between short-term and long-term exposure to $\mathrm{PM}_{10}$ and baPWV in apparently healthy subjects. With short-term exposure to $\mathrm{PM}_{10}$, non-obese subjects showed a marginally unfavorable association with baPWV. Further studies are necessary to validate and elucidate the mechanism underlying the effect of $\mathrm{PM}_{10}$ on baPWV.

Keywords: Vascular Stiffness; Air Pollution; Brachial-Ankle Pulse Wave Velocity; Particulate Matter

Received: July 28, 2020, Revised: October 11, 2020, Accepted: November 2, 2020

*Corresponding Author: Minseon Park https://orcid.org/0000-0001-6120-8356

Tel: +82-2-2072-3497, Fax: +82-2-766-3276, E-mail: msp20476@hanmail.net 


\section{INTRODUCTION}

According to the World Health Organization burden of disease from ambient air pollution reports, air pollution, especially that caused by particulate matter (PM), is a risk factor associated with acute lower respiratory infections, chronic obstructive pulmonary disease, lung cancer, ischemic heart disease (IHD), and stroke. ${ }^{1,2)}$ Ambient fine PM $\left(\mathrm{PM}_{2.5}\right)$ air pollution caused $7.6 \%$ of total mortality and contributed to $17.1 \%$ of IHD mortality in $2015 .{ }^{1)}$ Several mechanisms assessing the association between PM and cardiovascular disease have been proposed, but the association between PM and pulse wave velocity (PWV), which is an independent predictor of cardiovascular outcome, has not been fully investigated in the healthy population. One possible mechanism is that PM could be associated with endothelial dysfunction, accelerate acute arterial vasoconstriction in healthy adults, and eventually disrupt systolic function and increase heart rate variability. ${ }^{3)}$

Aortic PWV is an independent predictor of cardiovascular morbidity and mortality in patients with end-stage renal disease and essential hypertension as well as in the general population. ${ }^{4)}$ In addition, brachial-ankle PWV (baPWV) is an accurate and noninvasive indicator of aortic PWV and has been proven suitable for screening vascular damage in a large cohort. ${ }^{5}$ Among several studies evaluating the effects of PM on arterial stiffness, three studies focused on the effect of $\mathrm{PM}_{10}$. Adamopoulos et al. ${ }^{6}$ analyzed atmospheric pollution variables, including atmospheric PM concentrations, and their effects on arterial stiffness. They showed that 24-hour mean $\mathrm{PM}_{10}$ levels were associated with an increased amplitude of the aortic-wave reflection magnitude (augmentation pressure) in patients with hypertension but not with PWV. In a cross-sectional study of 127 patients undergoing hemodialysis with a mean baPWV of $1,767 \mathrm{~cm} / \mathrm{s}$, Weng et al. ${ }^{3)}$ reported that the 12-month average $\mathrm{PM}_{10}$ concentration $\left(57.9 \pm 5.7 \mu \mathrm{g} / \mathrm{m}^{3}\right)$ was associated with elevated baPWV. Ljungman et al. ${ }^{7)}$ showed that living near a major road was associated with higher arterial stiffness. However, satellite measurements of PM levels were not associated with arterial stiffness in the healthy Framingham Heart Study population, and no associations were observed with short-term exposure to air pollutants such as $\mathrm{PM}_{2.5}$, black carbon, sulfate, nitrogen oxides, and ozone.

Therefore, this study aimed to determine whether the correlation between long- and short-term exposure to $\mathrm{PM}_{10}$ and baPWV exists in apparently healthy cancer-free adults aged 40 years and older and to identify susceptible subgroups.

\section{METHODS}

\section{Study Population}

We initially investigated cancer-free patients who underwent medical screening examinations at Seoul National University Health Promotion Center between January 1, 2006, and December 31, 2009. Since the reference values of baPWV are influenced by age, participants aged 40 years and older were included in this study. Medical and demographic data were collected from chart reviews and online databases at our hospital. Patients with missing variables, such as blood pressure, fasting blood glucose, cholesterol levels (including high-density lipoprotein [HDL] cholesterol and triglycerides), waist circumference, body mass index (BMI), smoking status, drinking status, regular exercise, residence location, baPWV measurements, and antihypertensive and antidiabetic medication status were excluded from the study (Figure 1).

This study was conducted in accordance with the tenets of the Declaration of Helsinki and the need to obtain patient consent was waived by the Seoul National University Hospital Institutional Review Board (IRB approval no., E-1803-108-932).

\section{Major Exposure Variables}

Air pollution levels were recorded using a network of 265 monitoring stations near the patients' living areas in South Korea. Data on air quality status obtained by the Korean Ministry of Environment were used. ${ }^{8)}$ For $\mathrm{PM}_{10}$ values, the 24-hour average concentrations of PM on the day of medical screening and moving averages of the prior 1-3 days $\left(\mathrm{PM}_{10}\right.$ Lag 0 to $\mathrm{PM}_{10}$ Lag 0-3) were used to evaluate the effect of short-term exposure to $\mathrm{PM}_{10}$ on health outcomes. Long-term exposure to $\mathrm{PM}_{10}$ was examined using a 365-day moving average of $\mathrm{PM}_{10}$ concentrations $\left(\mathrm{PM}_{10} \mathrm{Lag} 0-365\right)$. If there was no monitoring station in a participant's residential district, we selected the pollution level from the nearest station.

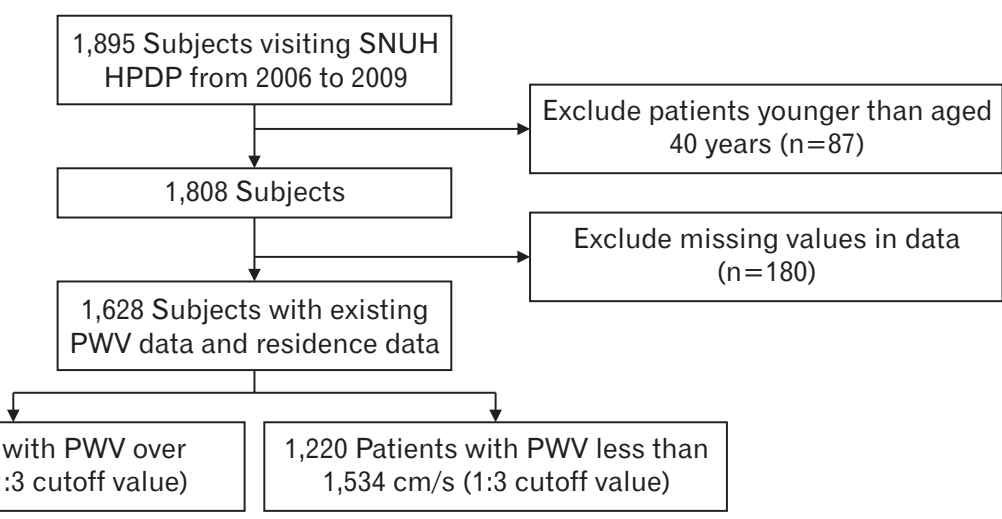

Figure 1. Inclusion and exclusion criteria of the eligible study population. SNUH, Seoul National University Hospital; PWV, pulse wave velocity; HPDP, Health Promotion Disease Prevention Center. 


\section{Covariates}

Hypertension and diabetes status, including the medication details, were ascertained using self-administered questionnaires. Patients were classified as nonsmokers, ex-smokers, and current smokers using self-administered structured questionnaires, which were rechecked by trained nurses. Heavy alcohol consumption was defined as alcohol consumption of more than $30 \mathrm{~g} / \mathrm{d}(210 \mathrm{~g} / \mathrm{wk}){ }^{9)}$ Concise methods for body composition measurements have been described previously. ${ }^{10)}$

\section{Definition of Metabolic Syndrome}

Metabolic syndrome was defined according to the National Cholesterol Education Program Adult Treatment Panel III (NCEP-ATP III) and American Heart Association/National Heart Lung and Blood Institute (AHA/NHLBI). ${ }^{11,12)}$ The abdominal obesity criterion was modified according to Asian guidelines. ${ }^{13)}$ As a result, patients who met three or more of the following criteria were selected: (1) waist circumference $\geq 90 \mathrm{~cm}$ for men or $\geq 80 \mathrm{~cm}$ for women; (2) triglyceride levels of $150 \mathrm{mg}$ / $\mathrm{dL}$ or higher; (3) HDL cholesterol levels less than $40 \mathrm{mg} / \mathrm{dL}$ in men or less than $50 \mathrm{mg} / \mathrm{dL}$ in women; (4) blood pressure levels of 130/85 mm $\mathrm{Hg}$ or higher; and (5) elevated fasting blood sugar levels of $100 \mathrm{mg} / \mathrm{dL}$ or higher.

\section{Brachial-Ankle Pulse Wave Velocity Measurement}

BaPWV was measured using a Vascular Profiler 1000 (VP-1000; Omron
Healthcare, Kyoto, Japan), with blood pressure cuffs placed around the arms and legs in the supine position, and was calculated using the equation: baPWV=(D1-D2)/t, where D1 is the distance between the heart and ankle, D2 is the distance between the heart and brachium, and $t$ is the transit time between brachial arterial waves and tibial arterial waves. The baPWV was measured by a trained technician.

\section{Statistical Analysis}

Previously, a baPWV cutoff value of $1,547 \mathrm{~cm} / \mathrm{s}$, which is close to the highest quartile value of the outcome variable of baPWV $(=1,534 \mathrm{~cm} / \mathrm{s})$ in this study, has been suggested as an independent predictor of asymptomatic coronary artery disease evaluated by coronary computed tomography angiography. Considering previous studies, we used a cutoff value of $1,534 \mathrm{~cm} / \mathrm{s}$ for our data analysis.

Subjects were categorized into two groups: those less than the third quartile and those greater than or equal to the third quartile of baPWV $(1,534 \mathrm{~cm} / \mathrm{s}, 75$ th percentile). P-values were calculated using the t-test for continuous variables and chi-square test for categorical variables in the baPWV subgroups ( $<$ or $\geq 1,534 \mathrm{~cm} / \mathrm{s}$ ). Univariate and multivariate logistic regression analyses were performed using a baPWV cutoff value of $1,534 \mathrm{~cm} / \mathrm{s}$ as an outcome variable and $\mathrm{PM}_{10}$ as exposure variables (short- and long-term), including age, sex, the use of antihypertensive medication and anti-diabetic medication, systolic blood pressure, daily mean temperature, year of examination, and residence area

Table 1. Baseline characteristics according to baPWV cutoff of $1,534(\mathrm{~cm} / \mathrm{s})$

\begin{tabular}{|c|c|c|c|c|}
\hline Characteristic & Total & $P W V \geq 1,534(\mathrm{~cm} / \mathrm{s})$ & $\mathrm{PWV}<1,534(\mathrm{~cm} / \mathrm{s})$ & $\mathrm{P}$-value \\
\hline No. of patients & 1,628 & 408 & 1,220 & \\
\hline Age (y) & $56.0 \pm 8.4$ & $62.1 \pm 8.3$ & $54.0 \pm 7.5$ & $<0.001$ \\
\hline Male & $862(53.0)$ & $229(56.1)$ & $633(51.9)$ & 0.13 \\
\hline Body mass index $\left(\mathrm{kg} / \mathrm{m}^{2}\right)$ & $24.4 \pm 3.1$ & $24.7 \pm 3.0$ & $24.3 \pm 3.1$ & 0.01 \\
\hline Diabetic medication & $146(9.0)$ & $75(18.4)$ & $71(5.8)$ & $<0.001$ \\
\hline Hypertensive medication & $438(26.9)$ & $181(44.4)$ & $257(21.1)$ & $<0.001$ \\
\hline Heavy alcohol drinking & $139(8.5)$ & $29(7.1)$ & $110(9.0)$ & 0.23 \\
\hline Smoking status & & & & 0.15 \\
\hline Never smoker & $932(57.2)$ & $232(56.9)$ & $700(57.4)$ & \\
\hline Ex-smoker & $436(26.8)$ & $121(29.7)$ & $315(25.8)$ & \\
\hline Current smoking & $260(16.0)$ & 55 (13.5) & 205 (16.8) & \\
\hline Systolic BP (mm Hg) & $130.0 \pm 16.1$ & $141 \pm 14.4$ & $125.7 \pm 14.7$ & $<0.001$ \\
\hline Diastolic BP (mm Hg) & $78.2 \pm 10.6$ & $83.7 \pm 10.6$ & $76.3 \pm 9.9$ & $<0.001$ \\
\hline Cholesterol & $203.9 \pm 35.6$ & $205.6 \pm 34.6$ & $203.4 \pm 35.9$ & 0.27 \\
\hline High-density lipoprotein (mg/dL) & $54.6 \pm 13.4$ & $53.2 \pm 13.2$ & $55.0 \pm 13.5$ & 0.02 \\
\hline Glucose $(\mathrm{mg} / \mathrm{dL})$ & $95.6 \pm 20.5$ & $103.4 \pm 26.5$ & $93.0 \pm 17.3$ & $<0.001$ \\
\hline Metropolitan living & $1,007(61.9)$ & $270(66.2)$ & $737(60.4)$ & 0.04 \\
\hline Capital living & 866 (53.2) & $231(56.6)$ & $635(52.1)$ & 0.11 \\
\hline Average daily temperature $\left({ }^{\circ} \mathrm{C}\right)$ & $13.9 \pm 9.5$ & $13.4 \pm 9.8$ & $14.1 \pm 9.4$ & 0.18 \\
\hline \multicolumn{5}{|l|}{ Lag days of $\mathrm{PM}_{10}\left(\mu \mathrm{g} / \mathrm{m}^{3}\right)$} \\
\hline $\operatorname{Lag} 0$ & $57.3 \pm 32.2$ & $58.2 \pm 33.2$ & $57.0 \pm 31.9$ & 0.52 \\
\hline Lag 0-1 & $55.9 \pm 28.9$ & $56.8 \pm 29.9$ & $55.7 \pm 28.5$ & 0.49 \\
\hline Lag 0-2 & $55.2 \pm 26.7$ & $55.5 \pm 26.4$ & $55.1 \pm 26.8$ & 0.79 \\
\hline Lag 0-3 & $54.6 \pm 24.9$ & $54.4 \pm 23.8$ & $54.6 \pm 25.3$ & 0.88 \\
\hline Lag 0-365 & $57.7 \pm 6.5$ & $57.4 \pm 6.5$ & $57.8 \pm 6.5$ & 0.24 \\
\hline
\end{tabular}

Values are presented as mean \pm standard deviation for continuous variables and or number (\%) for categorical variables.

baPWV, brachial-ankle pulse wave velocity; BP, blood pressure; $\mathrm{PM}_{10}$, particulate matter with an aerodynamic diameter of $10 \mu \mathrm{m}$ or less; $\mathrm{PM}_{10} \mathrm{Lag}_{0-365,1-y e a r}$ average $\mathrm{PM}_{10}$ level; $\mathrm{PM}_{10} \mathrm{Lag} 0,24$-hour average $\mathrm{PM}_{10}$ on the day of health examination; $\mathrm{PM}_{10} \mathrm{Lag} 0-n$, average $\mathrm{PM}_{10}$ levels on the concurrent day and $\mathrm{n}$ previous days. 
of participants as covariates. An effect modification analysis was conducted to investigate vulnerable subgroups by including an interaction term of $\mathrm{PM}_{10}$ exposure and a subgroup in addition to the covariates in the main models. The odds ratios (ORs) and 95\% confidence intervals were estimated. Statistical analysis was performed using Stata ver. 16.0 (Stata Corp., College Station, TX, USA).

\section{RESULTS}

A total of 1,628 patients were eligible for the study, as shown in Figure 1. Table 1 shows the baseline characteristics of the study population stratified according to a baPWV cutoff of 1,534 cm/s. The mean \pm standard deviation baPWV and age of the total study population were $1,419 \pm 244$ $\mathrm{cm} / \mathrm{s}$ and $56.0 \pm 8.4$ years, respectively.

In the study population, age, hypertensive medication, diabetes

Table 2. Logistic regression analysis with baPWV cutoff $1,534 \mathrm{~cm} / \mathrm{s}$ as the dependent variable $(\mathrm{N}=1,628)$

\begin{tabular}{|c|c|c|c|c|}
\hline Variable & Unadjusted OR (95\% Cl) & P-value & Adjusted OR $(95 \% \mathrm{Cl})^{*}$ & P-value \\
\hline Age & $1.13(1.12-1.15)$ & $<0.001$ & $1.13(1.11-1.15)$ & $<0.001$ \\
\hline Male & $1.19(0.95-1.49)$ & 0.14 & $0.99(0.78-1.26)$ & 0.95 \\
\hline BMl & $1.05(1.01-1.09)$ & 0.01 & $1.08(0.95-1.23)$ & 0.41 \\
\hline Systolic BP & 1.07 (1.06-1.08) & $<0.001$ & $1.08(1.07-1.09)$ & $<0.001$ \\
\hline Diastolic BP & 1.07 (1.06-1.09) & $<0.001$ & $1.10(1.08-1.12)$ & $<0.001$ \\
\hline Triglycerides & $1.002(1.001-1.003)$ & 0.002 & $1.002(1.001-1.004)$ & $<0.001$ \\
\hline Cholesterol & $1.002(0.999-1.005)$ & 0.27 & $1.005(1.001-1.009)$ & 0.01 \\
\hline High-density lipoprotein & $0.99(0.98-1.00)$ & 0.02 & $0.99(0.98-1.00)$ & 0.19 \\
\hline Glucose & $1.02(1.02-1.03)$ & $<0.001$ & $1.02(1.01-1.02)$ & $<0.001$ \\
\hline \multicolumn{5}{|l|}{ Lag days of $\mathrm{PM}_{10}$} \\
\hline $\operatorname{Lag} 0$ & 1.001 (0.998-1.005) & 0.52 & $1.002(0.998-1.006)$ & 0.36 \\
\hline $\operatorname{Lag} 0-1$ & 1.001 (0.997-1.005) & 0.50 & $1.002(0.998-1.007)$ & 0.36 \\
\hline Lag 0-2 & $1.001(0.996-1.005)$ & 0.79 & $1.001(0.996-1.006)$ & 0.76 \\
\hline $\operatorname{Lag} 0-3$ & $1.000(0.995-1.004)$ & 0.88 & $1.000(0.994-1.005)$ & 0.88 \\
\hline Lag 0-365 & $0.990(0.973-1.007)$ & 0.24 & $0.996(0.969-1.023)$ & 0.75 \\
\hline
\end{tabular}

baPWV, brachial-ankle pulse wave velocity; $\mathrm{OR}$, odds ratio; $\mathrm{Cl}$, confidence interval; $\mathrm{BMl}$, body mass index; $\mathrm{BP}$, blood pressure; $\mathrm{PM}_{10}$, particulate matter with an aerodynamic diameter of $10 \mu \mathrm{m}$ or less; $\mathrm{PM}_{10} \mathrm{Lag}$ 0-365, 1-year average of $\mathrm{PM}_{10}$ level; $\mathrm{PM}_{10}$ Lag 0, 24-hour average of $\mathrm{PM}_{10}$ on the day of health examination; $P M_{10}$ Lag 0-n, average $\mathrm{PM}_{10}$ levels on the concurrent day and $\mathrm{n}$ previous days.

${ }^{*}$ ORs are expressed as per $1 \mu \mathrm{g} / \mathrm{m}^{3}$ increase in average residential PM $\mathrm{PM}_{10}$ concentration. Logistic regression was done for predictors and baPWV cutoff $1,534 \mathrm{~cm} / \mathrm{s}$ which are categorical variables. In adjusted models, adjustment was done for age, sex, BMI, district (region), systolic BP, average daily temperature, hypertensive medication, diabetes medication, and year of examination.

Table 3. The association of $\mathrm{PM}_{10}$ Lag 0-365 (long-term exposure to $\mathrm{PM}_{10}$ ) with baPWV in subgroups according to clinical risk factors, demographics, and laboratory findings

\begin{tabular}{lccc}
\hline \multicolumn{1}{c}{ Variable } & & \multicolumn{2}{c}{ PWV cutoff $1,534 \mathrm{~cm} / \mathrm{s}$} \\
& Over PWV cutoff/subgroup population & OR $(95 \% \mathrm{Cl})$ & P-value \\
\hline Age $\geq 65 \mathrm{y}$ & $160 / 278$ & $0.961(0.902-1.023)$ & 0.21 \\
Age $<65 \mathrm{y}$ & $248 / 1,350$ & $1.004(0.972-1.037)$ & 0.82 \\
Male & $229 / 862$ & $1.001(0.962-1.042)$ & 0.95 \\
Female & $179 / 766$ & $0.988(0.943-1.035)$ & 0.61 \\
BMl $\geq 25.0 \mathrm{~kg} / \mathrm{m}^{2}$ & $181 / 639$ & $0.991(0.948-1.036)$ & 0.69 \\
BMl $<25.0 \mathrm{~kg} / \mathrm{m}^{2}$ & $227 / 989$ & $1.001(0.961-1.042)$ & 0.97 \\
With hypertension & $181 / 438$ & $0.985(0.935-1.038)$ & 0.55 \\
No hypertension & $227 / 1,190$ & $0.991(0.954-1.028)$ & 0.62 \\
With diabetes & $75 / 146$ & $1.076(0.957-1.211)$ & 0.22 \\
No diabetes & $333 / 1,482$ & $0.987(0.957-1.018)$ & 0.41 \\
Never smoker & $232 / 932$ & $1.014(0.974-1.056)$ & 0.50 \\
Ex-smoker & $121 / 436$ & $0.986(0.934-1.041)$ & 0.62 \\
Current smoker & $55 / 260$ & $0.955(0.873-1.045)$ & 0.32 \\
Regular exercise & $195 / 720$ & $1.009(0.963-1.057)$ & 0.72 \\
Not regular & $213 / 908$ & $0.982(0.944-1.021)$ & 0.33 \\
Metabolic syndrome & $182 / 454$ & $0.971(0.926-1.019)$ & \\
No metabolic & $226 / 1,174$ & $1.006(0.967-1.046)$ & 0.24 \\
\hline
\end{tabular}

Adjustment was done for age, sex, BMl, district (region), systolic blood pressure, average daily temperature, hypertensive medication, diabetes medication, and year of examination.

$\mathrm{PM}_{10}$, particulate matter with an aerodynamic diameter of $10 \mu \mathrm{m}$ or less; $\mathrm{PM}_{10}$ Lag 0-365, 1-year average of PM ${ }_{10}$ level; baPWV, brachial-ankle pulse wave velocity; PWV, pulse wave velocity; $\mathrm{OR}$, odds ratio; $\mathrm{Cl}$, confidence interval; $\mathrm{BMI}$, body mass index. 
mellitus medication status, blood pressure (both systolic and diastolic), and glucose levels were statistically different between the high $(\geq 1,534)$ and low $(<1,534)$ baPWV groups. More specifically, patients with high baPWV were mostly older, were more likely to take hypertensive medication, had higher blood pressure, used more diabetes medication, and had higher glucose levels. There were no statistically significant differences between $\mathrm{PM}_{10}$ levels at Lag 0 to Lag 3 days and in moving averages (Table 1).

Table 2 also shows the statistically significant associations between age, systolic and diastolic blood pressure, triglyceride levels, glucose levels, and high baPWV on unadjusted logistic regression analysis. However, we did not observe a significant relationship between shortand long-term exposure to $\mathrm{PM}_{10}$ and high baPWV in the total study population. Although there were no significant associations between long-term exposure to $\mathrm{PM}_{10}$ and high baPWV in the subgroups (Table 3), the associations between short-term exposure to $\mathrm{PM}_{10}$ and high baPWV were marginally significant in non-obese subjects (Figure 2). A 10 - $\mu$ g increase in the 24-hour moving average at Lag 0-1 $\mathrm{PM}_{10}$ exposure was associated with a $6 \%$ increased risk of high baPWV in non- obese subjects (OR, 1.059; $\mathrm{P}=0.058$ ). The elderly, non-obese, and hypertensive individuals showed statistically non-significantly higher ORs than that did their counterparts (the young, obese and non-hypertensive individuals) during short-term exposure to $\mathrm{PM}_{10}$ in Lag 0-1. When we conducted stratified analysis to determine the differential effects of short-term exposure to $\mathrm{PM}_{10}$ exposure of Lag 0 to Lag 0-3 on high PWV, an unfavorable effect was more prominent in the nonobese group than in the obese group (P-value for interaction $\mathrm{PM}_{10} \mathrm{Lag}$ 0, 0.041; $\mathrm{PM}_{10}$ Lag 0-1, 0.038) (Supplementary Table 1, Figure 2).

\section{DISCUSSION}

When we assessed the short-term $\mathrm{PM}_{10}$ exposure through the 24-hour moving average of Lag 0 to Lag 3 days prior to medical examination, we found no statistically significant associations in the total study population and subgroups. When we investigated the subgroups according to age, sex, BMI, anti-hypertensive and anti-diabetes medication status, and metabolic syndrome with short-term exposure to $\mathrm{PM}_{10}$ from Lag 0 to Lag 3, participants with a lower BMI $\left(<25.0 \mathrm{~kg} / \mathrm{m}^{2}\right)$ pre-
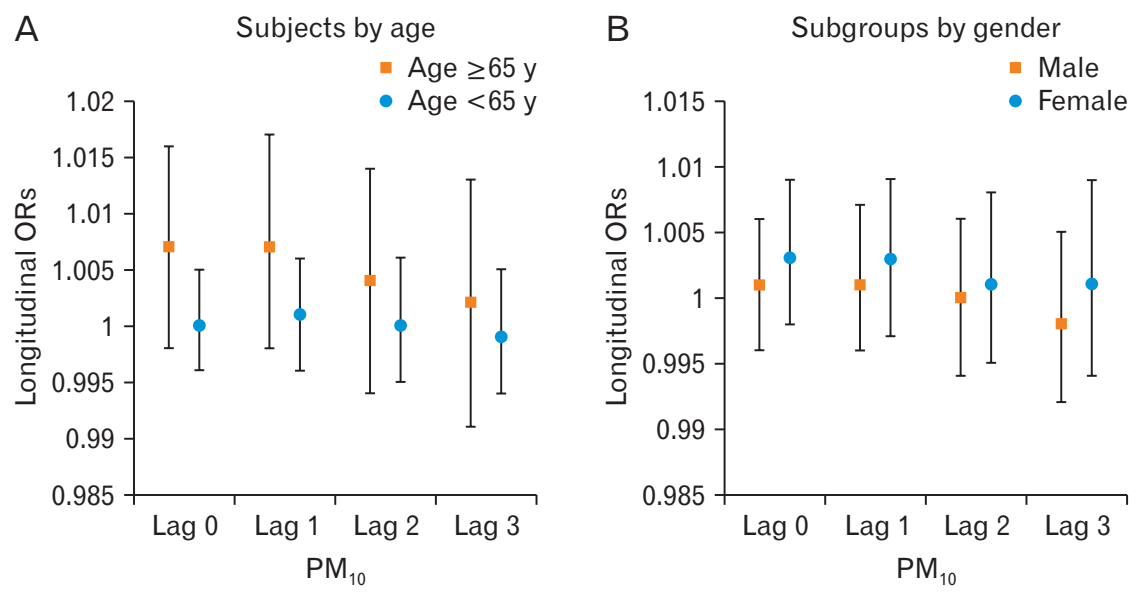

E

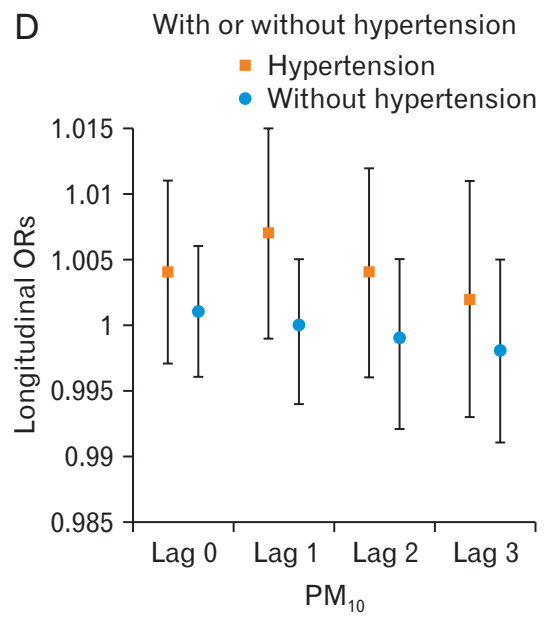

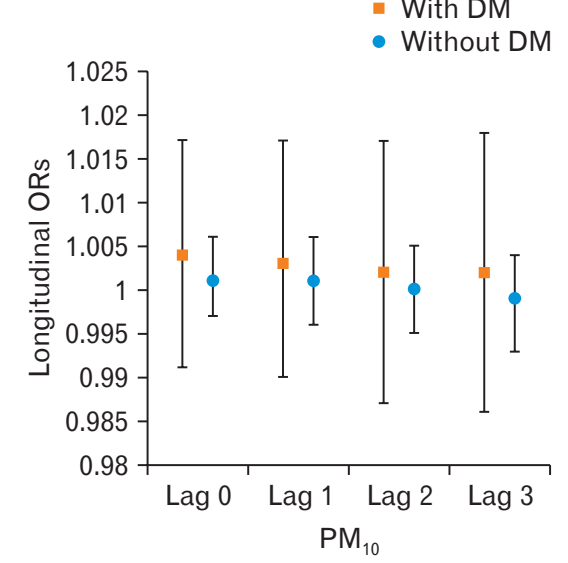

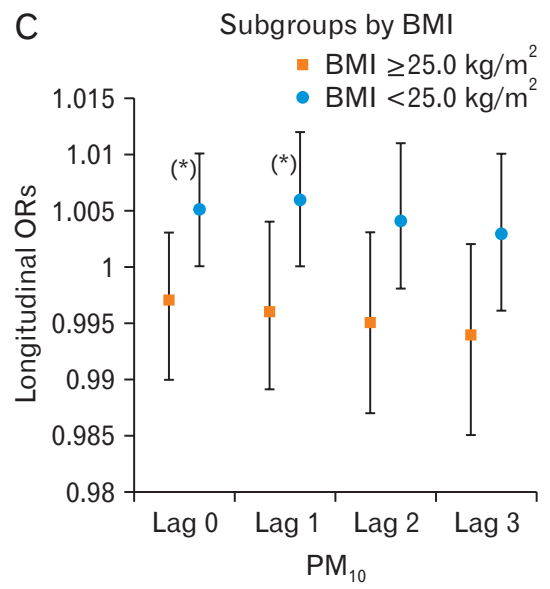

$\mathrm{F}$

With or without MetS

- With MetS

- Without MetS

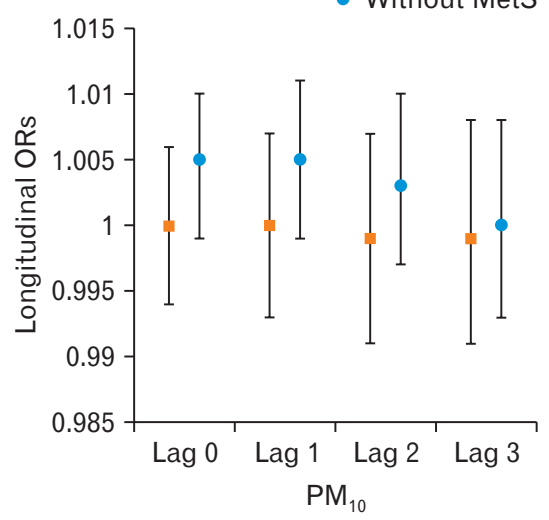

Figure 2. Acute effect of $\mathrm{PM}_{10}$ on brachial-ankle pulse wave velocity, according to the subgroups of age (A), gender (B), body mass index (BMI) (C), hypertension (D), diabetes mellitus (DM) status (E), and metabolic syndrome (MetS) (F). The PM10 moving averages are shown on the $x$-axis. *Asterisks indicate statistically significant differences between subgroups with $95 \%$ confidence intervals (P-value for interaction $<0.05)$. Adjustments were made for age, sex, BMl, district (region), systolic blood pressure, average daily temperature, hypertensive medication, DM medication, and year of examination. $\mathrm{PM}_{10}$, particulate matter with an aerodynamic diameter of $10 \mu \mathrm{m}$ or less; $\mathrm{OR}$, odds ratio. 
sented a slightly greater risk of high PWV compared to their counterparts with a moving average of Lag $0-1$. In particular, a 10- $\mu$ g increase in the 24-hour moving average of $\mathrm{PM}_{10}$ exposure of Lag 0-1 was marginally associated with a $6 \%$ elevated risk of high baPWV in non-obese subjects. Long-term exposure assessed through a 365-day moving average of $\mathrm{PM}_{10}\left(\mathrm{PM}_{10}\right.$ Lag 0-365) was not associated with an increased risk of high baPWV among all subjects and subgroups.

Previous studies on the association between $\mathrm{PM}_{10}$ and baPWV showed inconsistent results due to differences in study populations and small sample sizes. In a systematic review, based on the published literature until January 2017, Zanoli et al. ${ }^{14)}$ suggested that short- and long-term exposure to gaseous and PM pollutants, including nitric oxide, sulfur dioxide, $\mathrm{PM}_{10}$, and $\mathrm{PM}_{2.5}$, may be associated with increased arterial stiffness and wave reflection. As a potential marker of arterial stiffness, parameters such as carotid-femoral PWV, baPWV, and augmentation index were assessed in 11 published studies from 2010 to 2016. Various subgroups such as older adults, urban population, patients with hypertension, and patients undergoing hemodialysis were more sensitive to air pollution variables, as manifested by arterial stiffness. ${ }^{3,6,15,16)}$ Among them, only one study conducted by Weng et al. ${ }^{3)}$ observed that the 12-month average concentration of $\mathrm{PM}_{10}$ was associated with elevated baPWV in patients undergoing hemodialysis $(\beta=0.13, P=0.04)$.

In 2018, in a well-characterized large community-based cohort of the Framingham Heart study, ${ }^{7)}$ a positive association between living near a major road and higher arterial stiffness was observed. However, no associations were observed with short- and long-term exposure to air pollutants such as the satellite-measured levels of $\mathrm{PM}_{2.5}$, black carbon, sulfate, nitrogen oxides, and ozone, with arterial stiffness. Recently, an interquartile increase in $\mathrm{PM}_{10}$ and $\mathrm{PM}_{2.5}$ did not significantly increase the PWV in 40 healthy volunteers aged 60 years and older in a randomized, crossover study. ${ }^{17)}$ Our study also showed no significant associations between short-term and long-term exposure to $\mathrm{PM}_{10}$ and baPWV in apparently healthy cancer-free subjects. We observed a marginally elevated risk of high PWV associated with short-term exposure to a 24-hour moving average of $\mathrm{PM}_{10}$ exposure of Lag 0-1 in nonobese subjects. This association in non-obese subjects was significantly different from that in obese subjects.

Several mechanisms underlying the association between PM and PWV have been postulated. Mild and transitory inflammatory stimuli have been proposed as possible links between arterial stiffening, ${ }^{14)}$ functional arterial stiffening, and short-term exposure to air pollutants. Additionally, exposure to PM pollutants is associated with acute arterial vasoconstriction ${ }^{18)}$ and endothelial dysfunction. ${ }^{19)}$ Long-term exposure to PM for months to years has been associated with atherosclerosis, vascular dysfunction, and eventually subclinical diseases such as high blood pressure, increased carotid intima media thickness, and increased calcification of arteries. ${ }^{7)}$

The possible mechanism underlying the differential effect of shortterm exposure to $\mathrm{PM}_{10}$ on high PWV in non-obese subjects may be as follows. Previously, Tang et al. ${ }^{20)}$ suggested that obese individuals have greater nutritional reserves and can therefore cope better with acute stressful events and increased metabolic demands due to the beneficial effect of adipose tissue induced by beneficial hormones and cytokines. In our study, relatively healthy, non-obese individuals showed significantly lower body fat percentage based on our data (body fat \% of obese versus non-obese $=30.4 \%$ versus $25.9 \%$ ), which may be a possible mechanism by which non-obese individuals have difficulty coping with short-term exposure to $\mathrm{PM}_{10}$, resulting in high PWV. Further research is required to validate our findings.

This study used various laboratory data and clinical measurements to determine specific vulnerable subgroups and explain the potential links underlying the relationship between PM and inflammation, fitness, level of adiposity, and PWV. However, our study had some limitations. Although we analyzed the association between $\mathrm{PM}_{10}$ and baPWV in short- and long-term exposures, we did not analyze $\mathrm{PM}_{2.5}$, because $\mathrm{PM}_{2.5}$ data were only available for the city of Seoul between 2006 and 2009, whereas $\mathrm{PM}_{10}$ was available for the entire nation. The study population we used underwent private health screening tests in a single university hospital; thus, this group may not be representative of the general population. Furthermore, we used both short- and long-term PM exposure assessments with fixed monitoring data, which may cause non-differential misclassification at the individual level of exposure and underestimate the association in long-term exposure-related adverse health effects. Finally, because we did not have information regarding the timing of the activities of each patient, we could not ascertain the exact location and timing of exposure to $\mathrm{PM}_{10}$, which could lead to a bias if the $\mathrm{PM}_{10}$ levels between the patient's workplace and residence differ. Nevertheless, because exposure misclassification could lead to a null hypothesis, we can presume that our results could underestimate the effects of $\mathrm{PM}_{10}$ compared to those that could be obtained by measuring $\mathrm{PM}_{10}$ exposure levels with more precision. In other words, because we did not install 24-hour air pollution monitoring devices for each patient, we could not consider the air pollution levels when commuting to and from work; thus, a more sophisticated modeling approach is required in future studies.

In conclusion, we showed the probable adverse effect of short-term exposure to $\mathrm{PM}_{10}$ on high baPWV in non-obese subjects compared to their obese counterparts. Further large and prospective studies are necessary to validate and elucidate the mechanism underlying of the influence of PM on baPWV, especially in healthy, non-obese subjects.

\section{CONFLICT OF INTEREST}

No potential conflict of interest relevant to this article was reported.

\section{SUPPLEMENTARY MATERIALS}

Supplementary materials can be found via https://doi.org/10.4082/ kjfm.20.0180. Supplementary Table 1. Differential effect of short-term exposure of $\mathrm{PM}_{10}$ on brachial-ankle pulse wave velocity, according to age, gender, BMI, hypertension, diabetes status, and MetS, all with 
$\mathrm{PM}_{10}$ moving averages.

\section{ORCID}

Young Jun Park: https://orcid.org/0000-0001-6045-7406

Yu Jin Cho: https://orcid.org/0000-0002-3183-6607

Jinseul Kwak: https://orcid.org/0000-0001-8253-4687

Youn-Hee Lim: https://orcid.org/0000-0002-1290-5814

Minseon Park: https://orcid.org/0000-0001-6120-8356

\section{REFERENCES}

1. Cohen AJ, Brauer M, Burnett R, Anderson HR, Frostad J, Estep K, et al. Estimates and 25-year trends of the global burden of disease attributable to ambient air pollution: an analysis of data from the Global Burden of Diseases Study 2015. Lancet 2017;389:1907-18.

2. GBD 2015 Risk Factors Collaborators. Global, regional, and national comparative risk assessment of 79 behavioural, environmental and occupational, and metabolic risks or clusters of risks, 1990-2015: a systematic analysis for the Global Burden of Disease Study 2015. Lancet 2016;388:1659-724.

3. Weng CH, Hu CC, Yen TH, Huang WH. Association between environmental particulate matter and arterial stiffness in patients undergoing hemodialysis. BMC Cardiovasc Disord 2015;15:115.

4. Meaume S, Benetos A, Henry OF, Rudnichi A, Safar ME. Aortic pulse wave velocity predicts cardiovascular mortality in subjects $>70$ years of age. Arterioscler Thromb Vasc Biol 2001;21:2046-50.

5. Yamashina A, Tomiyama H, Takeda K, Tsuda H, Arai T, Hirose K, et al. Validity, reproducibility, and clinical significance of noninvasive brachial-ankle pulse wave velocity measurement. Hypertens Res 2002;25:359-64.

6. Adamopoulos D, Vyssoulis G, Karpanou E, Kyvelou SM, Argacha JF, Cokkinos D, et al. Environmental determinants of blood pressure, arterial stiffness, and central hemodynamics. J Hypertens 2010;28:903-9.

7. Ljungman PL, Li W, Rice MB, Wilker EH, Schwartz J, Gold DR, et al. Long- and short-term air pollution exposure and measures of arterial stiffness in the Framingham Heart Study. Environ Int 2018;121(Pt 1): $139-47$.

8. Kim SU, Kim KY. Physical and chemical mechanisms of the daily-toseasonal variation of $\mathrm{PM}_{10}$ in Korea. Sci Total Environ 2020;712: 136429 .
9. Traversy G, Chaput JP. Alcohol consumption and obesity: an update. Curr Obes Rep 2015;4:122-30.

10. Park M, Yoon E, Lim YH, Kim H, Choi J, Yoon HJ. Renal hyperfiltration as a novel marker of all-cause mortality. J Am Soc Nephrol 2015;26: 1426-33.

11. Benedict M. NCEP's new guidelines at a glance: National Cholesterol Education Program. Health Care Food Nutr Focus 2002;18:8-9.

12. Skilton MR, Moulin P, Serusclat A, Nony P, Bonnet F. A comparison of the NCEP-ATPIII, IDF and AHA/NHLBI metabolic syndrome definitions with relation to early carotid atherosclerosis in subjects with hypercholesterolemia or at risk of CVD: evidence for sex-specific differences. Atherosclerosis 2007;190:416-22.

13. Hong AR, Lim S. Clinical characteristics of metabolic syndrome in Korea, and its comparison with other Asian countries. J Diabetes Investig 2015;6:508-15.

14. Zanoli L, Lentini P, Granata A, Gaudio A, Fatuzzo P, Serafino L, et al. A systematic review of arterial stiffness, wave reflection and air pollution. Mol Med Rep 2017;15:3425-9.

15. Mehta AJ, Zanobetti A, Koutrakis P, Mittleman MA, Sparrow D, Vokonas $\mathrm{P}$, et al. Associations between short-term changes in air pollution and correlates of arterial stiffness: the Veterans Affairs Normative Aging Study, 2007-2011. Am J Epidemiol 2014;179:192-9.

16. Wu CF, Shen FH, Li YR, Tsao TM, Tsai MJ, Chen CC, et al. Association of short-term exposure to fine particulate matter and nitrogen dioxide with acute cardiovascular effects. Sci Total Environ 2016;569-570:3005.

17. Sinharay R, Gong J, Barratt B, Ohman-Strickland P, Ernst S, Kelly FJ, et al. Respiratory and cardiovascular responses to walking down a trafficpolluted road compared with walking in a traffic-free area in participants aged 60 years and older with chronic lung or heart disease and age-matched healthy controls: a randomised, crossover study. Lancet 2018;391:339-49.

18. Brook RD, Brook JR, Urch B, Vincent R, Rajagopalan S, Silverman F. Inhalation of fine particulate air pollution and ozone causes acute arterial vasoconstriction in healthy adults. Circulation 2002;105:1534-6.

19. Tamagawa E, Bai N, Morimoto K, Gray C, Mui T, Yatera K, et al. Particulate matter exposure induces persistent lung inflammation and endothelial dysfunction. Am J Physiol Lung Cell Mol Physiol 2008;295:L79-85.

20. Tang B, Luo F, Zhao J, Ma J, Tan I, Butlin M, et al. Relationship between body mass index and arterial stiffness in a health assessment Chinese population. Medicine (Baltimore) 2020;99:e18793. 